\title{
Normal integral bases for infinite abelian extensions
}

\author{
by \\ PATRIK LundSTRÖM (Göteborg)
}

1. Introduction. Let $K \supseteq \mathbb{Q}$ be a Galois field extension with Galois group $G$ and ring of algebraic integers $R$. We consider $G$ as a topological group with the Krull topology (see e.g. [L93, p. 329]).

Suppose that $K \supseteq \mathbb{Q}$ is a finite extension. The normal basis theorem asserts that there is $x \in K$ such that $B:=\{\sigma(x)\}_{\sigma \in G}$ form a basis for $K$ as a vector space over $\mathbb{Q}$. In fact, such a basis exists for general finite Galois field extensions (see e.g. [J80, p. 283]). $B$ is called a normal basis and $x$ is called a normal basis generator. If $B$ is a $\mathbb{Z}$-basis for $R$, then $B$ is called a normal integral basis and $x$ is called a normal integral basis generator. Normal integral bases do not always exist. In fact, for abelian extensions, Leopoldt [Leo59] proved the following result:

1.1. Theorem. Suppose that $K \supseteq \mathbb{Q}$ is a finite Galois field extension with abelian Galois group. Then $K$ has a normal integral basis if and only if $K$ is contained in the $n$th cyclotomic number field, $K_{n}$, for some positive square-free integer $n$.

For infinite extensions Theorem 1.1 makes no sense. However, if we let $(G, \mathbb{Z})$ denote the set of functions $f: G \rightarrow \mathbb{Z}$ and we let $G$ operate on $(G, \mathbb{Z})$ by $(\sigma f)(\tau)=f\left(\sigma^{-1} \tau\right)$ for $\sigma, \tau \in G$, then Theorem 1.1 can be formulated by saying that there is a left $\mathbb{Z}$-module isomorphism $F:(G, \mathbb{Z}) \rightarrow R$ that respects the action of $G$.

Namely, if $B$ is a $\mathbb{Z}$-basis for $R$, then we can define $F$ by

$$
F(f)=\sum_{\sigma \in G} f(\sigma) \sigma(x) .
$$

Conversely, if $F:(G, \mathbb{Z}) \rightarrow R$ is an isomorphism as above, and $h: G \rightarrow \mathbb{Z}$ is defined by $h(1)=1$ and $h(\sigma)=0, \sigma \neq 1$, then $x:=F(h)$ is a normal integral basis generator for $K$. In this paper we prove, using an idea introduced by Lenstra in [Le85] for the case of normal bases for infinite Galois field

2000 Mathematics Subject Classification: 11R04, 11R18, 11R33. 
extensions, that this version of Theorem 1.1 is valid for infinite extensions, provided we only consider continuous functions $G \rightarrow \mathbb{Z}$ :

1.2. Theorem. Suppose that $K \supseteq \mathbb{Q}$ is a Galois field extension with abelian Galois group $G$ and ring of algebraic integers $R$. Denote by $C(G, \mathbb{Z})$ the $\mathbb{Z}$-module of all continuous functions $f: G \rightarrow \mathbb{Z}$, where $\mathbb{Z}$ is equipped with the discrete topology. Let $G$ operate on $C(G, \mathbb{Z})$ by $(\sigma f)(\tau)=f\left(\sigma^{-1} \tau\right)$ for $\sigma, \tau \in G$. Then there is an isomorphism of $\mathbb{Z}$-modules $C(G, \mathbb{Z}) \rightarrow R$ that respects the action of $G$ if and only if for every finite extension $K^{\prime} \supseteq \mathbb{Q}$ such that $K \supseteq K^{\prime}$ there is a positive square-free integer $n$ such that $K_{n} \supseteq K^{\prime}$.

For some related results concerning normal bases for infinite Galois extensions see [Lu98] and [Lu99].

2. Cofinal countable inverse limits. We recall the following definitions. A set $I$ is preordered if it is equipped with a binary relation $\prec$ that is transitive and reflexive. A set $I$ is directed if it is preordered and has the additional property that for any two $\alpha, \beta \in I$ there is $\gamma \in I$ such that $\alpha \prec \gamma$ and $\beta \prec \gamma$. An inverse system of sets $\left(E_{\alpha}, f_{\alpha \beta}\right)$ relative to a set $I$ consists of a preordered set $I$, a set $E_{\alpha}$ for each $\alpha \in I$, and a map $f_{\alpha \beta}: E_{\beta} \rightarrow E_{\alpha}$ for each pair $\alpha, \beta \in I$ with $\alpha \prec \beta$, such that $f_{\alpha \alpha}=\operatorname{id}_{E_{\alpha}}$ for each $\alpha \in I$, and $f_{\alpha \beta} f_{\beta \gamma}=f_{\alpha \gamma}$ for all $\alpha, \beta, \gamma \in I$ with $\alpha \prec \beta \prec \gamma$. The inverse limit of such a system, denoted by $\lim _{\alpha \in I} E_{\alpha}$, is defined to be the set of all $\left(x_{\alpha}\right)_{\alpha \in I}$ in $\prod_{\alpha \in I} E_{\alpha}$ such that if $\alpha, \beta \in I$ and $\alpha \prec \beta$, then $f_{\alpha \beta}\left(x_{\beta}\right)=x_{\alpha}$. Recall that a subset $J$ of $I$ is called cofinal if for every $\alpha \in I$ there is $\beta \in J$ such that $\alpha \prec \beta$. We use the following result in Section 3:

2.1. Proposition. Let $\left(E_{\alpha}, f_{\alpha \beta}\right)$ be an inverse system of sets relative to a directed set $I$, which has a countable cofinal subset $J$. Suppose furthermore that all $f_{\alpha \beta}, \alpha, \beta \in J$, are surjective. If all $E_{\alpha}$ are non-empty, then the inverse limit $\lim _{\alpha \in I} E_{\alpha}$, taken with respect to the maps $f_{\alpha \beta}, \alpha, \beta \in I$, is non-empty.

Proof. Use the ideas in [B68, III.7.4, Prop. 5].

3. Number fields. In this section, we prove Theorem 1.2. We need three well known results (see Lemmas 3.1-3.3). The multiplicative group of units of a ring $S$ is denoted by $S^{*}$.

3.1. Lemma. Let $L^{\prime} \supseteq L$ be finite Galois field extensions of $\mathbb{Q}$. Let $\operatorname{Tr}$ denote the trace map from $L^{\prime}$ to $L$. Suppose that $L \supseteq \mathbb{Q}$ has Galois group $H$.

(a) If $L$ has a normal integral basis, then $\mathbb{Z}[H]^{*}$ acts transitively on the set of normal integral basis generators for $L$.

(b) If $x$ is a normal integral basis generator for $L^{\prime}$, then $\operatorname{Tr}(x)$ is a normal integral basis generator for $L$. 
Proof. (a) follows directly from the definition of a normal integral basis generator and (b) is [N90, Theorem 4.10].

Let $\left\{p_{1}, p_{2}, \ldots\right\}$ denote the set of all odd primes. For each $p_{i}$, let $\varepsilon_{p_{i}}$ denote a primitive $p_{i}$ th root of unity. For all positive integers $m, n$ such that $m \geq n$, let

$$
r_{n}^{m}: \operatorname{Gal}\left(K_{p_{1} \ldots p_{m}} / \mathbb{Q}\right) \rightarrow \operatorname{Gal}\left(K_{p_{1} \ldots p_{n}} / \mathbb{Q}\right)
$$

be the restriction map and let

$$
p_{n}^{m}: \mathbb{Z}_{p_{1}-1} \times \ldots \times \mathbb{Z}_{p_{m}-1} \rightarrow \mathbb{Z}_{p_{1}-1} \times \ldots \times \mathbb{Z}_{p_{n}-1}
$$

be the natural projection. For each positive integer $n$, let

$$
\theta_{n}: \mathbb{Z}_{p_{1}-1} \times \ldots \times \mathbb{Z}_{p_{n}-1} \rightarrow \operatorname{Gal}\left(K_{p_{1} \ldots p_{n}} / \mathbb{Q}\right)
$$

be the group isomorphism given by $\theta_{n}\left(a_{1}, \ldots, a_{n}\right)=\sigma_{a_{1}, \ldots, a_{n}}$, where

$$
\sigma_{a_{1}, \ldots, a_{n}}\left(\varepsilon_{i}\right)=\varepsilon_{i}^{a_{i}}, \quad i=1, \ldots, n .
$$

With the above notations, we immediately get:

3.2. Lemma. If $m$ and $n$ are positive integers such that $m \geq n$, then the following diagram is commutative:

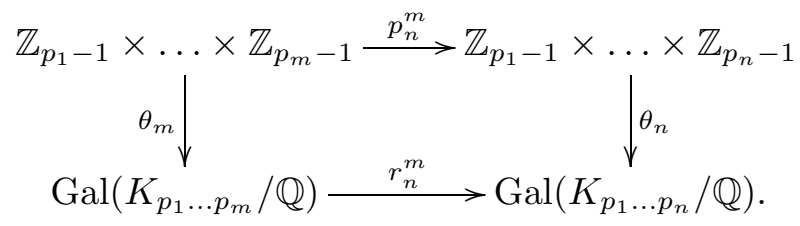

We also need the following:

3.3. Lemma. If $G_{1}$ and $G_{2}$ are groups, then the map

$$
p: \mathbb{Z}\left[G_{1} \times G_{2}\right]^{*} \rightarrow \mathbb{Z}\left[G_{2}\right]^{*},
$$

induced by the projection $G_{1} \times G_{2} \rightarrow G_{2}$, is surjective.

Proof. The inclusion $p\left(\mathbb{Z}\left[G_{1} \times G_{2}\right]^{*}\right) \subseteq \mathbb{Z}\left[G_{2}\right]^{*}$ is trivial. For the reverse inclusion, let $i: \mathbb{Z}\left[G_{2}\right]^{*} \rightarrow \mathbb{Z}\left[G_{1} \times G_{2}\right]^{*}$ be the map induced by the canonical injection $G_{2} \rightarrow G_{1} \times G_{2}$. Then $\mathbb{Z}\left[G_{2}\right]^{*}=p\left(i\left(\mathbb{Z}\left[G_{2}\right]^{*}\right)\right) \subseteq p\left(\mathbb{Z}\left[G_{1} \times G_{2}\right]^{*}\right)$.

Proof of Theorem 1.2. Let $U$ denote the set of open subgroups of $G$. If $N \in U$ let

$$
K^{N}=\{k \in K \mid \sigma(k)=k \text { for all } \sigma \in N\} .
$$

We write $N^{\prime} \prec N$ when $N, N^{\prime} \in U$ and $N \subseteq N^{\prime}$.

Assume that there is an isomorphism of $\mathbb{Z}$-modules $F: C(G, \mathbb{Z}) \rightarrow R$ that respects the action of $G$. Pick a finite field extension $K^{N} \supseteq \mathbb{Q}$, where $N \in U$. By Theorem 1.1, it is enough to show that $K^{N}$ has a normal integral basis. Let $C_{N}(G, \mathbb{Z})=\{f \in C(G, \mathbb{Z}) \mid \sigma f=f$ for all $\sigma \in N\}$. Then 
$F\left(C_{N}(G, \mathbb{Z})\right)=R^{N}$. If we define $h \in C_{N}(G, \mathbb{Z})$ by $h(\sigma)=1$ if $\sigma \in N$ and $h(\sigma)=0$ if $\sigma \notin N$, then $F(h)$ is a normal integral basis generator for $K^{N}$.

Now suppose that if $K^{\prime} \supseteq \mathbb{Q}$ is a finite extension such that $K \supseteq K^{\prime}$, then there is a positive integer $n$ such that $K_{n} \supseteq K^{\prime}$. We can assume that $K \supseteq K_{n}$ for all positive square-free integers $n$. By Theorem 1.1, for every $N \in U$ there is a normal integral basis generator $y_{N}$ for $K^{N}$. If $N^{\prime} \prec N$, then let $\operatorname{Tr}_{N^{\prime} / N}: K^{N} \rightarrow K^{N^{\prime}}$ denote the trace function and define $\beta_{N^{\prime} / N} \in$ $\mathbb{Z}\left[G / N^{\prime}\right]^{*}$ by the relation $\operatorname{Tr}_{N^{\prime} / N}\left(y_{N}\right)=\beta_{N^{\prime} / N}\left(y_{N^{\prime}}\right)$. This is possible because of Lemma 3.1(a), (b). If $N^{\prime} \prec N$, then let $\varrho_{N^{\prime} / N}: \mathbb{Z}[G / N] \rightarrow \mathbb{Z}\left[G / N^{\prime}\right]$ denote the natural map and define the function $\gamma_{N^{\prime} / N}: \mathbb{Z}[G / N]^{*} \rightarrow \mathbb{Z}\left[G / N^{\prime}\right]^{*}$ by $\gamma_{N^{\prime} / N}\left(\alpha_{N}\right)=\varrho_{N^{\prime} / N}\left(\alpha_{N}\right) \beta_{N^{\prime} / N}$ for all $\alpha_{N} \in \mathbb{Z}[G / N]^{*}$. It is easy to check that $\left(\mathbb{Z}[G / N]^{*}, \gamma_{N^{\prime} / N}\right)$ form an inverse system of sets relative to $U$. Let

$$
V=\left\{N \in U \mid K^{N}=K_{p_{1} \ldots p_{n}} \text { for some } n \geq 1\right\} .
$$

By Lemmas 3.2 and 3.3, the functions $\gamma_{N^{\prime} / N}, N, N^{\prime} \in V$, are surjective. Since $V$ is a countable cofinal subset of $U$, we see, by Proposition 2.1, that the inverse limit $\Gamma:=\lim _{N \in U} \mathbb{Z}[G / N]^{*}$ taken with respect to the functions $\gamma_{N^{\prime} / N}$, is non-empty. Now choose $\left(\alpha_{N}\right)_{N \in U} \in \Gamma$. For every $N \in U$, let $x_{N}=\alpha_{N}\left(y_{N}\right)$. Then, by Lemma 3.1(a) and the above construction, we get:

(i) if $N \in U$, then $x_{N}$ is a normal integral basis generator for $K^{N}$,

(ii) if $N^{\prime} \prec N$, then $\operatorname{Tr}_{N^{\prime} / N}\left(x_{N}\right)=x_{N^{\prime}}$.

Let $f \in C(G, \mathbb{Z})$. Since $G$ is compact and $\mathbb{Z}$ is equipped with the discrete topology, there is $N \in U$ such that $f$ is constant on $\tau N$ for every choice of $\tau \in G$. We can therefore define a map $f_{N}: G / N \rightarrow \mathbb{Z}$ induced by $f$. We now define $F: C(G, \mathbb{Z}) \rightarrow R$ by

$$
F(f)=\sum_{\sigma \in G / N} f_{N}(\sigma) \sigma\left(x_{N}\right) .
$$

By (ii), $F$ is well defined. It is clear that $F$ is $\mathbb{Z}$-linear. By (i), $F$ is bijective. It is easy to check that $F$ also respects the action of $G$.

\section{References}

[B68] N. Bourbaki, Set Theory, Hermann, 1968.

[J80] N. Jacobson, Basic Algebra I, Freeman, 1980.

[L93] S. Lang, Algebra, Addison-Wesley, 1993.

[Le85] H. W. Lenstra, Jr., A normal basis theorem for infinite Galois extensions, Indag. Math. 47 (1985), 221-228.

[Leo59] H.-W. Leopoldt, Über die Hauptordnung der ganzen Elemente eines abelschen Zahlkörpers, J. Reine Angew. Math. 201 (1959), 119-149.

[Lu98] P. Lundström, Self-dual normal bases for infinite Galois field extensions, Comm. Algebra 26 (1998), 4331-4341. 
[Lu99] P. Lundström, Normal bases for infinite Galois ring extensions, Colloq. Math. 79 (1999), 235-240.

[N90] W. Narkiewicz, Elementary and Analytic Theory of Algebraic Numbers, Springer, 1990.

Department of Mathematics

Chalmers University of Technology

and the University of Göteborg

S-412 96 Göteborg, Sweden

E-mail: lund@math.chalmers.se 